\title{
La política de educación integral en estados brasileños: \\ un análisis de su trayectoria
}

\author{
Jeffrey, Debora \\ Universidade Estadual de Campinas, Campinas, Brasil \\ debora.jeffrey@gmail.com
}

\section{Resumen}

La educación integral en Brasil como política nacional se ha configurado como una proposición y iniciativa de carácter pedagógico, que objetiva la ampliación de la jornada escolar, así como la formación integral de niños y jóvenes de la educación básica. El marco legal que fundamentó la propuesta de educación integral (Portaría Interministerial n. 17 de 24/04/2007 y Decreto $n^{0} 7.083$ de 27/01/2010) estableció que el Programa Más Educación fuera reconocido como una medida inductora para la adhesión de estados, municipios y Distrito Federal a política de educación integral en país. Por esto, el estudio analiza las tipologías de política de educación integral en los estados brasileños , con énfasis en alcance, la normalización, la formulación, la identificación del establecimiento de relaciones intersectoriales y el argumento de la política. Se evidenció que la reconfiguración del pacto y gobernanza federativa, tuve efecto directo en diseño, formulación, implementación de políticas y programas de educación integral o educación de tiempo integral, en estados brasileños.

\section{Abstract}

The integral education in Brazil as a national policy has been configured as a proposition and initiative of a pedagogical character, which aims to promote the expansion of the school day, as well as the integral formation of children and youth in basic education. The legal framework that mantain the integral education proposal (Interministerial Ordinance n. 17 of 24/04/2007 and Decree n. 7.083 of 27/01/ 2010) established that the More Education Program was recognized as an inductive measure for the adhesion of states, municipalities and Federal District for the integral education policy in the country. From this perspective, the study analyzes the typologies of integral education policy in brazilian states, with emphasis on covarege, normatization, formulation, identification of the establishment of intersectoral relations and the argument of politics. It was evidenced that the reconfiguration of the pact and federative governance, it had been a direct effect under the design, formulation, implementation of policy and programs related to integral education or full-time education, in brazilian states.

Palabras clave: Educación integral, Política Educativa, Governanza, Brasil.

Keywords: Integral Education. Educational Policy. Governance. Brazil.

\section{INTRODUCCIÓN}

La educación integral en Brasil se configuró como política, inicialmente, en una ordenación pedagógica pautada en la formación integral de niños y jóvenes de la educación básica a través de la ampliación de la jornada escolar. Su propuesta inicial, es parte de y una discusión que se centró en la reorganización de los espacios educativos, a partir de la introducción de las actividades extra-turno en las unidades escolares que participan en este proyecto.

El proceso de disposición de la educación integral no es nuevo. En Brasil, el debate se origina con el Manifiesto de los Pioneros de la Educación, publicado en 1932, con gran influenza de la ideología liberal, lo que represento un hito en las discusiones sobre el papel de las escuelas públicas para la formación integral de los ciudadanos en el diálogo con las transformaciones instituidas en la sociedad con el advenimiento de la República, en un escenario cuya educación abarcaba sólo grupos específicos de los ciclos de poder. 
En este sentido, tratando de romper con una lógica en la que el acceso a la escuela y el proceso de formación individual estaba relacionado a su condición social, el Manifiesto de los Pioneros de la Educación, introdujo el debate sobre la manutención de una educación integral en los establecimientos públicos la educación.

Ante este marco, fueron diversas las experiencias en escuelas con modelos que buscaron garantizar la educación integral en el territorio nacional. En los últimos treinta años (los años 1980 y 1990), estos registros se potencian, la observación de casos como los Centros Integrados de Educación

Pública (CIEP ‘s), Gobierno Estatal de Leonel Brizola en el estado de Río de Janeiro (1983-1987); los Centros de Educación Integral para Niños y Adolescentes (CAIC), en Gobierno Presidencial de Fernando Collor de Melo (1990-1992); Ios Centros Educativos Unificados (CEU), Gobierno de la Municipalidad de Marta Suplicy en San Paulo (2001-2004); Programa Bairro Escuela Ciudad Educadora, en Gobierno de la municipalidad de Lindberg Farias en Nova Iguaçu - RJ (2005-2010); el Programa Integrado de la Escuela, el Gobierno de Municipalidad de Fernando Pimentel, en ciudad de Belo Horizonte (2006-2010) y el Programa de desarrollo y educación integral de Pernambuco - Escuela de referencia, del Gobernador Estatal Eduardo Henrique Accioly Campos (2007-2010).

En el proceso más reciente, vale mencionar el papel del Plan de Desarrollo de la Educación (PDE) para la consolidación de la política de educación integral en Brasil. Aprobado en 2007, el documento propone el Programa Más Educación entre sus acciones para el mejoramiento de la educación nacional. Con la propuesta de ampliación de la educación a tiempo integral en el país, el Programa es considerado como un inductor de la política pública de formación integral para las escuelas públicas brasileñas, en especial para la ampliación de la jornada escolar a siete horas de un día en las unidades escolares de la enseñanza primaria.

Articulado a propuesta Educación del tiempo integral, el Fondo de Mantenimiento y Desarrollo de la Educación Básica y Valorización de Profesionales de la Educación (FUNDEB) y el Plan Nacional de Educación (PNE), sancionada el 25 de junio de 2014, vinculó la asignación de recursos públicos para la educación integral, al prever el financiamiento para la implementación de la medida, asegurando la ampliación de jornada escolar.

\section{SOBRE LA EDUCACIÓN INTEGRAL EN BRASIL}

Sin embargo, mientras que la formación integral propuesta por Teixeira, en los años de 1930, no tuvo éxito debido a la falta de presupuestos, en los años 2000, las Organizaciones de la Sociedad Civil de Interés Público (OSCIP`s), actúan juntamente con los gobiernos estatales y locales con el fin de administrar fondos y ofrecer el asesoramiento técnico. Esa estrategia política fuera adoptada directamente con el Programa Más Educación, creado en el año 2007 y extinto el año 2017.

En consonancia con la legislación educativa vigente, el Programa Más Educación fue instituido como elemento fundamental para la ampliación de la jornada escolar. Formulado por el Gobierno Federal en asociación con los estados, las municipalidades, el Distrito Federal y las OSCIP's.

Así, sobre la participación de las OSCIP's en Programa Más Educación, es posible identificar que esas organizaciones promovieron una nueva forma de participación de las instituciones privadas -que constituyen y mantén grupos sin fines lucrativos, identificadas cómo sociedad civil organizada y con voz activa para proponer proyectos y programas, directamente relacionadas a política de educación integral en Brasil.

La participación de estas organizaciones sociales, sin ánimo de lucro, se puede observar a través de la difusión de mensajes de que la población escolar en situación de vulnerabilidad social, debe ser atendida por el Programa Más Educación, a través de programas y acciones que consisten en la experiencia a través de una educación integral, con el fin de combatir las desigualdades sociales y educativas (LECLERC, MOLL, 2012).

Otro aspecto que llama la atención es que dicho Programa permite la creación de un nuevo tipo de asociación entre el gobierno y el sector privado, que Olmedo (2013) llama de gobernanza filantrópica (Olmedo, 2013), ya que gran parte los participantes de las OSCIP's tienen los fines de difundir las propuestas de educación integral por medio de conglomerados corporativos. 


\section{EL PÚBLICO-PRIVADO COMO BASES PARA LA DIFUSIÓN DE LA POLÍTICA DE EDUCACIÓN INTEGRAL EN ESTADOS BRASILEÑOS}

Articulando el favorecimiento de la ampliación de la jornada escolar con la formación integral de niños y jóvenes de la educación básica, la política nacional de educación integral tiene como principal elemento el Programa Más Educación.

El Programa previa la creación de un Foro Inter-Ministerial y un Comité Metropolitano, con el fin de articular las acciones de los gobiernos (federal, estatal y municipal) y la sociedad civil organizada en relación al diseño, desarrollo e implementación de las propuestas de educación integral (BRASIL, 2014). A partir de esa observación, el equipo de investigadores del Grupo de Estudios, Pesquisas en Política y Evaluacion Educacional (GEPALE) ${ }^{1}$, de Universidad Estatal de Campinas, SP (Brasil), empezó sus estudios, con base na propuesta analítica de Parente (2016): tipología de educación integral, que orientó na analice de la categoría - tipo de relación que fuera establecida entre el Poder Público y el sector privado, a través de la implementación de la educación integral en los estados brasileños. La equipo delimitó que la analice de esta categoría empezaría con la identificación de las OSCIP's con participación con algún tipo de asesoría o participación junto a los estados brasileños en alguna actividad relacionada a política de educación integral.

El diseño de la investigación, dialoga con la discusión de Olmedo (2013), que comprende que el Estado y el mercado se articula a través de la creación de redes políticas, ya que este proceso implica en un proceso definido como de gobernabilidad filantrópica.

Así pues, combinando investigación en etapas según las indicaciones de Olmedo (2013), empezamos la busca en sitios de algunas OSCIP's con referencia a política de educación integral y apoyo al Gobierno estatal en Brasil. A partir de este estudio, los sitios de noticias relacionadas con estas instituciones fueron consultados con el fin de identificar: los tipos de acciones y asociaciones entre el Gobierno, e su espacio de acción.

En esto proceso, fueron recolectados datos, referentes al año 2017, y identificadas las OSCIP's:

- Instituto Ayrton Senna.

- Fundación Darcy Ribeiro.

- Educación y Participación.

- Fundación Lemann.

- Fundación Telefónica.

- Inspirare.

- Todos por la Educación.

- Itaú Social.

- Centro de Referencia para la Educación Integral.

- Centro de Estudios e Investigación en Educación, Cultura y Acción Comunitaria.

Inicialmente, la información que se encuentro correspondió a la obra de cada una de estas OSCIP's, sus acciones en los sistemas de educación pública estatal. La equipo de investigación concluyó que todas las organizaciones estaban a participar en al menos veinticuatro lugares en Brasil entre los estados y municipios, y en las cinco regiones administrativas del país (Norte, Sul, Sudeste, Nordeste y Centro- Oeste).

En un segundo paso, el procedimiento de refinamento de estos datos, la equipo de investigación delimitó la amostra de las OCIP's entre los estados brasileños y su relación directa con los Departamentos Estatales de Educación a través de proyectos o acciones en asociaciones para el desarrollo de la educación integral. En esta etapa, se alcanzó el total de 14 estados participantes de este tipo de iniciativas en el Norte, Noreste, Medio Oeste y el Sudeste.

\footnotetext{
${ }^{1}$ Las pesquisadoras: Profa. Dra. Debora Cristina Jeffrey, Profa. Ms. Carolina Machado D’Ávila, Lívia Moura, Mariane Bernardes, Jaqueline Camargo, Suelen Batista y Claudiane Bordon.
} 
Además, para refinar la búsqueda, fue necesario para llevar a cabo la intersección de la información obtenida de la nueva investigación, ahora en los sitios oficiales diarios y de las Asambleas Legislativas de los estados brasileiros, las leyes estatales de búsqueda que rigen el acuerdo de transacción entre las entidades públicas y, el proceso de implementación de la política de educación integral con asesoría de una OSCIP.

En este caso, esa búsqueda identificó que las OSCIP's, sobre la educación integral en los estados brasileños, actuaban en año de 2017, en actividades de asistencia en la preparación y aplicación de directrices para la educación integral en conjunto con los órganos centrales de los Departamentos de Educación de los estados y municipios, así como el apoyo para la elaboración de material didáctico en la orientación y la formación de los profesionales de la educación en la promoción de la tecnología educativa, el seguimiento de las propuestas educativas, entre otros proyectos de innovación para la aprendizaje.

La última etapa consistió en la sistematización de las instituciones que establecieron, en el año 2017, alianzas junto a las Secretarías Estaduales de Educación, en la efectividad de la política de educación integral. Al fine del levantamiento, la equipo de investigación identificó las seguintes OSCIP's:

Cuadro 1 - Lista de OSCIP's en colaboración con el Departamento de Educación del estado. (Año 2017 - Estados y regiones).

\begin{tabular}{|c|c|c|}
\hline Nombre & Estados & Regiones \\
\hline CAPES & SC & Sul \\
\hline Cenários Pedagógicos & $\mathrm{BA}, \mathrm{PE}$ & Nordeste \\
\hline CENPEC & $\mathrm{PA}, \mathrm{BA}, \mathrm{PE}, \mathrm{GO}$ & Norte, Nordeste, Centro-Oeste \\
\hline Centro de Referências em Educação Integral - CREI & $\mathrm{AL}, \mathrm{BA}$ & Nordeste \\
\hline $\begin{array}{l}\text { Centro Integrado de Estudos e Programas de } \\
\text { Desenvolvimento Sustentável - CIEDS }\end{array}$ & $\mathrm{BA}, \mathrm{PE}$ & Nordeste \\
\hline Educação e Participação & $\mathrm{RR}, \mathrm{PA}, \mathrm{GO}$ & Norte, Centro-Oeste \\
\hline Flasco Brasil & $\mathrm{BA}, \mathrm{PE}$ & Nordeste \\
\hline Fundação Bradesco & $\mathrm{PE}$ & Nordeste \\
\hline Fundação Itaú Social & PA, BA, PE, GO & Norte, Nordeste, Centro-Oeste \\
\hline Fundação Lemann & $\mathrm{SP}, \mathrm{PB}$ & Sudeste, Nordeste \\
\hline Fundação SMA & $\mathrm{BA}, \mathrm{PE}$ & Nordeste \\
\hline Fundação Vivo & SE & Nordeste \\
\hline Instituto Alana & $\mathrm{BA}, \mathrm{PE}$ & Nordeste \\
\hline $\begin{array}{l}\text { Instituto Aliança - Banco Interamericano de } \\
\text { Desenvolvimento }\end{array}$ & CE & Nordeste \\
\hline Instituto Ayrton Senna & CE, SC, RJ & Nordeste, Sul, Sudeste \\
\hline Instituto C\&A & $\mathrm{BA}, \mathrm{PE}$ & Nordeste \\
\hline Instituto de Co-Responsabilidade Social - ICE & $\begin{array}{l}\text { RO, TO, SE, GO, ES, CE, PI, } \\
M A, P B, P E, S P\end{array}$ & $\begin{array}{l}\text { Norte, Nordeste, } \\
\text { Centro-Oeste, Sudeste }\end{array}$ \\
\hline Instituto Inspirare & AL,BA, PB, PE, SE & Nordeste \\
\hline Instituto Natura & $\begin{array}{l}\text { RO, TO, BA, CE, MA, PB, PE, } \\
\text { SE, GO, SC, PE, SP }\end{array}$ & $\begin{array}{l}\text { Norte, Nordeste, } \\
\text { Centro-Oeste, }\end{array}$ \\
\hline Instituto Oi & $\mathrm{BA}, \mathrm{PE}$ & Nordeste \\
\hline Instituto Porvir & SP & Sudeste \\
\hline Instituto Rodrigo Mendes & $\mathrm{BA}, \mathrm{PE}$ & Nordeste \\
\hline Instituto Sonho Grande & $\mathrm{RO}, \mathrm{TO}, \mathrm{GO}$ & Norte, Centro-Oeste \\
\hline Instituto Telefônica & $\mathrm{AL}, \mathrm{SE}, \mathrm{PB}, \mathrm{ES}, \mathrm{MG}, \mathrm{PA}, \mathrm{SP}$ & Norte, Nordeste, Sudeste \\
\hline Movimento de Ação e Interação Social - MAIS & $\mathrm{BA}, \mathrm{PE}$ & Nordeste \\
\hline Parceiros da Educação & SP & Sudeste \\
\hline *26 Instituições & ${ }^{*}$ Exceto: AM, MT, MS, DF & $\begin{array}{l}\text { *Todas as regiões } \\
\text { contempladas }\end{array}$ \\
\hline
\end{tabular}

Fuente: elaborado por las autoras. 
Con los resultados, se observó que estas instituciones hagan su trabajo con presupuestos públicos, ese ato tiene contribuido para la expansión y el fortalecimiento de la educación integral de tiempo completo entre escuelas de los estados brasileños. Así, después de las analices de las directrices, las acciones y también de los programas desarrollados por esas equipos, encontramos un discurso común acerca de la calidad de educación integral: la satisfacción de las necesidades de los estudiantes, con protección a su total desarrollo.

El argumento de la transformación de la educación para el cuidado de los pobres como una forma de superar las desigualdades sociales ha sido potente para la inserción de las escuelas públicas de OSCIP. En ese sentido, también señalamos la recurrencia al estímulo de profesores y gestores que se unen a la comunidad para llevar el aprendizaje al espacio urbano con premios, además de realizar proyectos para expandir los espacios de aprendizaje con materiales interactivos sobre tendencias en área de la educación dirigidas a la formación de quienes tiene interés en poner en práctica.

Presentado ante el panorama, es importante que tenga en cuenta los avances en esta investigación con el fin para identificar la formalización de alianzas entre OSCIP's y los Departamentos Estatales de Educación a través de la promulgación de leyes que tienen que ver con el tema, que describen cómo las redes de política se ha configurado y la profundidad de la aplicación de la formación integral en Brasil.

\section{DISCUSIÓN Y CONCLUSIONES}

Con base el análisis de los tipos de política de educación integral en los estados brasileños, con el orden del modo de comprender su alcance, la identificación de algunos de los actores involucrados en el proceso, se observó que la aplicación de la educación integral como una política pública para la educación brasileña es centralizado, principalmente en la ampliación de la jornada escolar.

Las instituciones identificadas guían su intervención en el argumento de la formación completa de la persona. Sin embargo, dadas las acciones propuestas, parece que la aplicación de la educación integral conversa más con el tiempo del estudiante en la escuela; incluso a considerar una educación que va delante de su espacio de convivio.

Además, el discurso de estas instituciones destaca la actividad empresarial como la principal forma de asegurar la inclusión digital y la preparación para la ciudadanía, y su difusión. Es así, considerado como una mensaje relevante para la transformación de la educación como un camino hacia el éxito académico de los estudiantes y una escuela de calidad.

Lo más, el análisis del discurso que estas instituciones propagaran sobre el pensamiento de la educación como construcción colectiva, es contradictoria, pues sus mantenedores son miembros vinculados a los gran grupos empresariales y financieros, nacionales y globales, tales como bancos, los fabricantes de cosméticos y automóviles, entre otros sectores. Para ellos, los programas y proyectos que apoyan, son comprendidas como la respuesta para el combate de la crisis de la educación pública, con objetivo de combate a mala estructura administrativa, poco investimento y bajos indicadores de desarrollo educativo. Esta identificación lleva a la reflexión sobre los intereses reales de estas organizaciones en la agenda de la educación pública brasileña y la relación que establecen con los gobiernos, la observación de la construcción de las redes sociales y políticos (Mizruchi, 2006), a partir de la difusión de estas ideas.

Considerar la implementación de la política de educación integral en , entre los estados con la ampliación de la jornada escolar, teniendo como medida inductora el Programa Más Educación significa reflexionar sobre el hecho de que las instituciones presentadas en este trabajo, así como otras existentes que, por la necesidad del recorte, no compusieron este texto, tienen la facultad de promover una negociación política identificada como filantro -legislación (Olmedo, 2013): un proceso en que grandes nombres del mercado financiero y empresarial se insertan en los servicios públicos y prueban su eficiencia. Al que se puede entender, esta es una tendencia política en Brasil, con influenza directa para la trayectoria de educación integral en país. 


\section{REFERENCIAS BIBLIOGRÁFICAS}

Brasil.(2014). Lei no 13.005, de 25 de junho de 2014. Aprova o Plano Nacional de Educação - PNE e dá outras providências. Brasília, DF, 26 jun. <http://www.planalto.gov.br/ccivil_03/_ato2011-2014/2014/lei/l13005.htm>. Acesso em: 04 ago. 2017.

Leclerc, G; Fátima, E; Moll, J. (2012). Programa Mais Educação: avanços e desafios para uma estratégia indutora da Educação Integral e em tempo integral. Educar em Revista, n. 45, p.91-110, set. FapUNIFESP http://dx.doi.org/10.1590/ s0104-40602012000300007.<http://www.scielo.br/pdf/er/n45/07.pdf>.

Olmedo, A. (2013). Heterarquias e «governança filantrópica» global na Inglaterra: Implicações e controvérsias para o controle social das políticas sociais. Educação e Políticas em Debate, Uberlândia, v. 2, n. 2, p.470-498, jul - dez.

Parente, C. M. D. (2016). Construindo uma tipología das políticas de educação integral. Roteiro, [s.I.], v. 41, n. 3, p.563-586, 4 out. Universidade do Oeste de Santa Catarina [Digite texto] http://dx.doi.org/10.18593/r.v41i3.10601. Disponível em: <http://editora.unoesc.edu.br/index.php/roteiro/article/view/10601/pdf>. Acesso em: 20 abr. 2017. 